\title{
When numbers can be misleading: lithium induced irreversible neurotoxicity at therapeutic drug levels
}

\author{
Jyoti Aggarwal ${ }^{\circledR}$, Zainab Mehdi ${ }^{\infty}$, Baldeep Kaur ${ }^{*}$, Yuvraj Singh Cheema ${ }^{\circledR}$, Monica Gupta
}

Department of General Medicine, Government Medical College and Hospital, Sector 32, Chandigarh, India

\begin{abstract}
Received: 1 September 2021 Accepted: 28 November 2021

Published online: 7 December 2021

*Corresponding author: Baldeep Kaur, Assistant Professor; Address:

Department of General Medicine Level 4 D Block, Government Medical College and Hospital, Sector

32, Chandigarh. Pin 160030.

Tel: +918727001312;

Email: mailto:dr.baldeep.brar@gmail. com

Competing interests: None.

Funding information: None.

Citation: Aggarwal J, Mehdi Z, Kaur B, Cheema YS, Gupta M.When numbers can be misleading: lithium induced irreversible neurotoxicity at therapeutic drug levels. Journal of Emergency Practice and Trauma 2022; 8(1): 69-73. doi: 10.34172 jept.2021.36
\end{abstract}

\begin{abstract}
Objective: Lithium is a principal drug used in the treatment of bipolar disorder (BPD). Due to its narrow therapeutic index, serum levels need to be monitored regularly. In elderly patients with renal dysfunction lithium toxicity can develop paradoxically within the therapeutic range. This can lead to erroneous diagnosis and delayed treatment resulting in irreversible neurological sequelae as is described in our case.

Case Presentation: A 65-year-old hypertensive female, with a 7-year history of BPD presented with decreased oral intake since 5-7 days, followed by altered sensorium. Neurological examination revealed coarse tremors in bilateral upper and lower limbs with spasticity, hyperreflexia, bilateral knee clonus. Twenty-five days earlier, she was prescribed Lithium carbonate. On evaluation she was found to have chronic kidney disease. Serum lithium levels came out to be $1.18 \mathrm{mg} / \mathrm{dL}$ (borderline high). After ruling out other differentials, a diagnosis of lithium toxicity was considered and she underwent two sessions of hemodialysis (HD) leading to significant improvement in sensorium; however, the patient had persistent dysarthria, difficulty in walking and proximal myopathy predominantly in the lower limbs. Nerve conduction studies confirmed the presence of axonal neuropathy. These findings of peripheral neuropathy (both sensory and motor) were suggestive of SILENT (syndrome of irreversible lithium-effectuated neurotoxicity).

Conclusion: Unintended lithium toxicity can occur even at therapeutic levels especially in the elderlies owing to its narrow therapeutic window, complex pharmacokinetics and numerous drug interactions. Lithium can result in irreversible neurotoxicity including SILENT; therefore, a high level of suspicion is required to prevent such permanent disability. Keywords: Elderly, Toxicity, Lithium, Peripheral neuropathy
\end{abstract}

\section{Introduction}

Lithium is the principal drug in the treatment of bipolar disorder (BPD) with a special role in the management of acute mood episodes, prevention of suicides and as a prophylactic drug (1). Due to its narrow therapeutic index, it is advised to maintain therapeutic plasma levels between $0.6-1.2 \mathrm{mEq} / \mathrm{L}$, failure of which can lead to either sub-therapeutic dosing or lithium toxicity. Serum lithium concentration is influenced by various factors like age, drug interactions, renal functions and co-morbid conditions. We present a case of unintentional lithium toxicity in a 65-year-old hypertensive female on treatment with lithium carbonate for BPD where decreased oral intake due to depressive phase and acute impairment in renal functions led to decreased lithium excretion resulting in toxicity even at otherwise defined therapeutic plasma levels. The patient had a persistent neurological deficit even after 2 months. We also take this opportunity to discuss pathophysiology and treatment of different subtypes of lithium toxicity including SILENT (syndrome of irreversible lithium-effectuated neurotoxicity) with a special focus on complex pharmacokinetics of lithium.

\section{Case Presentation}

An elderly female with a known case of BPD for the last 7 years came to our emergency department with the history of decreased appetite and altered sensorium over a period of one week. Her BPD was first diagnosed in 2013 and she remained stable throughout, except for the two episodes of relapse into mania in 2015 and 2017. Present history revealed new inappropriate behavior, confusion and agitation with a progressive decline in consciousness leading to the emergency visit. At the presentation she had a blood pressure of $160 / 90 \mathrm{~mm}$ of $\mathrm{Hg}$, pulse rate of 78 per minute, temperature 37 degree Celsius and saturation $94 \%$, with breathing room air and random blood sugar of $98 \mathrm{mg} / \mathrm{dL}$. She appeared dehydrated and skin turgor was diminished. Neurological examination revealed 
coarse tremors in bilateral upper and lower limbs with tone increased in the lower limbs predominantly. She had bilateral knee clonus, hyperreflexia with flexor plantar response. Meningeal signs were absent and the rest of the systemic examination was unremarkable. She responded only to painful stimulus and made incomprehensible sounds. Eyes revealed a spontaneous upward gaze and any attempt to look laterally elicited horizontal nystagmus bilaterally.

When the drug history was explored it was found that the patient consumed Tab Lithium Carbonate (Sustained Release preparation) $450 \mathrm{mg}$ twice daily, 25 days ago for depressive episode together with venlafaxine $75 \mathrm{mg} / \mathrm{d}$, amoxapine $50 \mathrm{mg} / \mathrm{d}$, clonazepam $0.5 \mathrm{mg} / \mathrm{d}$ and zolpidem $10 \mathrm{mg} / \mathrm{d}$. Review of her past record showed that she had taken lithium in 2013 and 2017 as well for manic episodes. The patient also had hypertension from the past 10 years which was well controlled on tablet telmisartan $40 \mathrm{mg} / \mathrm{d}$ and amlodipine $5 \mathrm{mg} / \mathrm{d}$. During Psychiatric consultation, Bush Francis Catatonia rating scale was administered, lorazepam challenge test was unsuccessful and catatonia was ruled out.

In the setting of sub-acute deterioration in sensorium with examination and medication history as described above, a broad differential diagnosis of non-convulsive status epilepticus, serotonin syndrome, neuroleptic malignant syndrome, metabolic encephalopathy, infectious central nervous system pathologies, septic encephalopathy, cerebrovascular accident or drug toxicity were considered. In view of the history of significant drug intake and high suspicion for toxicity, serum lithium levels were ordered which came out to be $1.18 \mathrm{mg} / \mathrm{dL}$ (borderline high).

Meanwhile, a non-contrast computed tomography

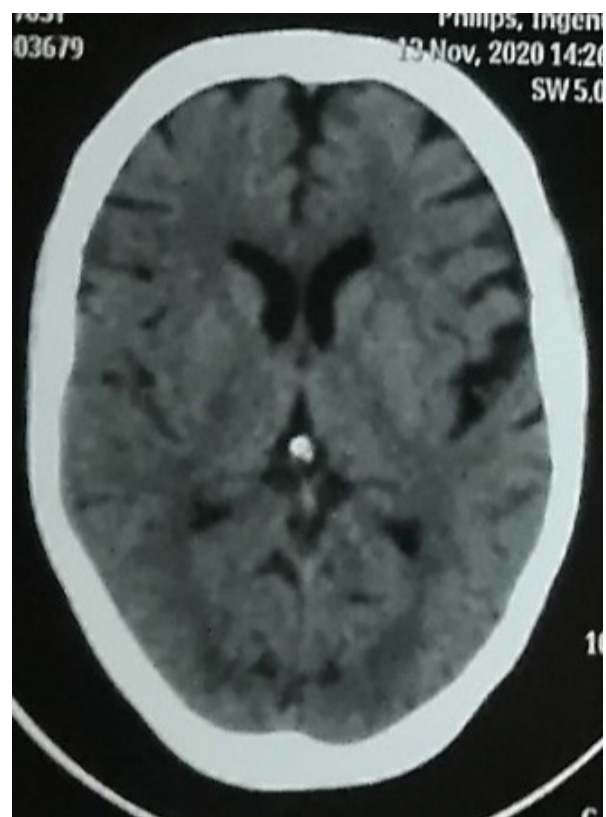

Figure 1. Non-contrast-enhanced CT brain showing cerebral atrophy with no parenchymal brain lesion. brain scan was performed which was grossly normal (Figure 1). Lumbar puncture for cerebrospinal fluid (CSF) examination was acellular with normal values of glucose $(106 \mathrm{mg} / \mathrm{dL})$, protein $(34 \mathrm{mg} / \mathrm{dL})$ and ADA (Nil). For other infectious etiologies blood, urine and CSF cultures were done which came out to be sterile. Magnetic Resonance Imaging of the brain was done which was grossly normal except for age related cerebral atrophy (Figure 2). EEG was suggestive of generalized cerebral dysfunction with no seizure activity.

Serial blood investigations were performed throughout her hospital admission as shown in Table 1. Renal function tests were deranged and arterial blood gas (ABG) had metabolic acidosis. Baseline ECG done on the first day of admission showed sinus rhythm and there was no evidence of peaked T waves, widening of QRS complex, prolonged PR interval or changes within the ST segment with corrected QT (QTc) of 360 milliseconds (normal $<450$ $\mathrm{msec}$ ). Ultrasound whole abdomen revealed grade 2 fatty liver, small sized left kidney $(6 \mathrm{~cm})$ with partially lost corticomedullary differentiation and bilateral renal parenchymal disease.

After ruling out various other causes, a diagnosis of lithium toxicity was made keeping in view the renal dysfunction leading to decreased excretion of lithium. Toxicity management for this patient included volume replacement for dehydration. In addition, the patient met EXTRIP criteria for hemodialysis (HD) and underwent the first course of $\mathrm{HD}$ on the $5^{\text {th }}$ day of admission after the opinion of a nephrologist followed by slight improvement in sensorium, but deteriorated again for which she underwent the second session of HD after 2 days leading to significant improvement in sensorium. However, she had persistent dysarthria, difficulty in walking and proximal myopathy predominantly in lower

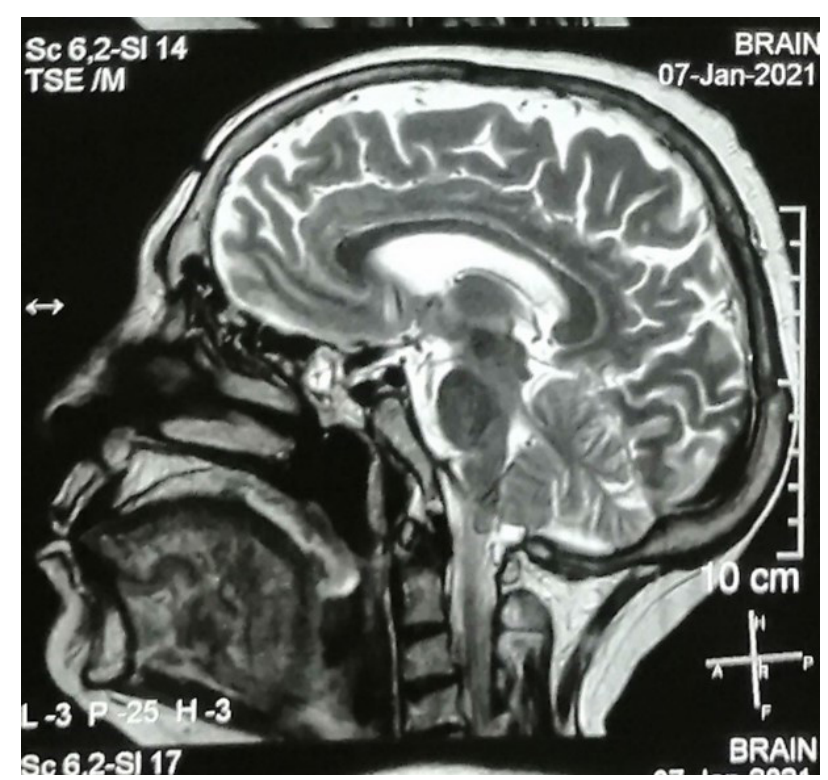

Figure 2. MRI brain with sagittal T2-weighted image showing age related brain atrophy. 
Table 1. Laboratory investigation during hospital stay

\begin{tabular}{|c|c|c|c|c|c|c|}
\hline & D- 1 & D- 3 & D-5 & D-8 (Post HD) & DAY 12 & At discharge \\
\hline Hemoglobin (g/dL) & 10.8 & 11.0 & 11.1 & 10.9 & 11.0 & 11.0 \\
\hline $\operatorname{TLC}\left(\times 10^{\wedge} 9 / \mathrm{L}\right)$ & 11.9 & 17.1 & 15.2 & 11.0 & 10.8 & 9.0 \\
\hline Platelet count $\left(\times 10^{\wedge} 9 / \mathrm{L}\right)$ & 337 & 354 & 350 & 327 & 331 & 308 \\
\hline Sodium & 148 & 147 & 144 & 131 & 140 & 147 \\
\hline Potassium & 4.6 & 5.1 & 5.4 & 4.1 & 4.7 & 3.9 \\
\hline Chloride & 109 & 115 & 116 & 100 & 104 & 108 \\
\hline Urea & 49 & 91 & 145 & 132 & 104 & 88 \\
\hline Creatinine & 1.8 & 2.1 & 2.3 & 1.8 & 1.6 & 1.7 \\
\hline Uric acid & 11.9 & 13.8 & 12.7 & 11.0 & 6.6 & \\
\hline Calcium & 8.9 & 9.0 & 9.1 & 8.2 & 8.8 & \\
\hline Phosphorus & 4.9 & 7.1 & 5.9 & 6.6 & 6.3 & \\
\hline $\operatorname{ALP}(30-150 \mathrm{U} / \mathrm{L})$ & 371 & & & & & 160 \\
\hline Arterial blood gas analysi & & & & & & \\
\hline $\mathrm{pH}$ & 7.290 & 7.320 & & & & \\
\hline $\mathrm{HCO}^{-}$ & 16.7 & 17.0 & & & & \\
\hline pCO2 & 35.2 & 33.2 & & & & \\
\hline pO2 & 85.5 & 106 & & & & \\
\hline Base excess & -8.6 & -7.6 & & & & \\
\hline Thyroid function tests & \multicolumn{6}{|c|}{ Within range } \\
\hline Urine R/E & \multicolumn{6}{|c|}{ Albumin $3+$, sugar- absent, pus cells $0-1$ and RBC $1-2$ /hpf } \\
\hline
\end{tabular}

TLC, total leucocyte count; ALP, alkaline phosphatase

limbs. Patient was discharged with normal serum lithium levels $(0.50 \mathrm{mg} / \mathrm{dL})$, but she was put on regular follow up for these persistent neurological symptoms. Nerve conduction studies were performed after two months which confirmed the presence of axonal neuropathy with a generalized decrease in amplitude of compound motor action potentials and sensory nerve action potential. There was no evidence of demyelinating neuropathy and hence a nerve biopsy was not performed. These findings of peripheral neuropathy (both sensory and motor) were suggestive of SILENT in this patient.

\section{Discussion}

Lithium for therapeutic use is available in immediate release (peak serum levels occurring at 2 to 4 hours) and sustained release preparations (peak levels delayed by 1.44 to 4.25 hours) (2). Once absorbed, lithium is slowly redistributed from the intravascular space to the total body water. Lithium has a three-compartment model of distribution, which aids in understanding current management and clearance of lithium as is shown in Figure 3 (3).

Lithium toxicity can be acute (as in suicidal poisoning), acute on chronic and chronic as in patients on lithium therapy with decreased renal function. When a patient on maintenance lithium therapy ingests large amounts all at once, acute on chronic toxicity manifests (4). In acute toxicity, gastrointestinal and cardio-toxic effects predominate while neurological involvement is delayed as opposed to chronic toxicity where neurological features dominate (5).

Neurological involvement in acute overdose may occur if kidney functions are significantly compromised, leading to redistribution of sufficient lithium from the intravascular to tissue compartments before it can be excreted (3). Such a scenario may arise when there is preexisting renal compromise, volume depletion secondary to gastrointestinal losses, sodium depletion or acute on chronic lithium toxicity. We presume this pathophysiology in our case as there was history of decreased oral intake with impaired renal function. In addition, the coprescription of telmisartan might have resulted in drug interactions with lithium. Other significant interactions have been reported with nonsteroidal anti-inflammatory

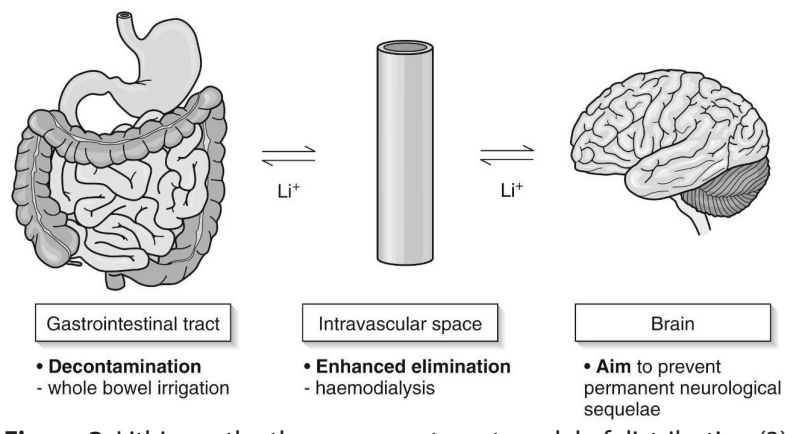

Figure 3. Lithium - the three-compartment model of distribution (3). 
drugs, angiotensinogen converting enzyme inhibitors, thiazides, spironolactone, diltiazem and verapamil leading to decreased lithium excretion through various mechanisms (6). When depletion of salt and water occurs in conditions like diarrhea, vomiting, reduced oral intake, there is increased sodium reabsorption in proximal convoluted tubule along with increased lithium uptake, which may cause acute intoxication (3). Volume depletion irrespective of origin can cause chronic lithium intoxication and fluid replacement helps reversal by maximizing lithium clearance (5).

We assert on the fact that such rare cases of lithium toxicity have been reported in patients with normal serum concentrations, sometimes labeled as lithium supersensitivity' or 'lithium-related idiosyncratic reaction' (7). With already established occurrence of renal impairment and serum lithium levels of $>0.8 \mathrm{mmol} / \mathrm{L}$, questions have been raised to reconsider the desired therapeutic levels in these patients.

Serum concentrations of lithium in elderlies are associated with reduced volume of distribution and reduced renal clearance in presence of co-morbidities like hypertension, congestive heart failure or renal dysfunction. Some case reports in older subjects indicate that lithium toxicity can occur at moderate blood levels of $0.5-0.8 \mathrm{mEq} / \mathrm{L}$. A case report by Foulser et al with a well-chosen title "Do not treat the numbers: lithium toxicity" described a case of a 62-year-old man with renal impairment taking $800 \mathrm{mg}$ lithium every night for the treatment of mania showed coarse, bilateral postural tremors and slurred speech with a lithium level of 0.95 $\mathrm{mmol} / \mathrm{L}$ (8). Peng described a case of an 18-year-old girl who was treated for acute mania with lithium carbonate $250 \mathrm{mg}$ twice a day and presented with loss of appetite, disturbed consciousness, anuria with a lithium blood level of $0.57 \mathrm{mmol} / \mathrm{L}$ i.e. at the lower end of the therapeutic range and was managed successfully with intravenous mannitol and immediate stopping of drug (9). Strayhorn et al reported 36 such cases of lithium toxicity with normal serum levels, 10 of whom had lithium concentrations $<1.1$ $\mathrm{mmol} / \mathrm{L}(10)$.

In 1980s it was first instituted that neurotoxicity with lithium is not merely an acute complication and for symptoms persisting beyond 2 months, a new term was coined -SILENT (11). In a review by Adityanjee et al of 90 cases of patients with persistent deficits the clinical manifestations varied from cerebellar dysfunction, extrapyramidal syndrome, brainstem dysfunction or dementia with varying organic mental syndromes to atypical presentations such as downbeat nystagmus, retrobulbar optic neuritis, persistent papilledema, choreoathetoid movements, peripheral neuropathy (both motor and sensory), myopathy and blindness due to central pontine myelinolysis (12). Our patient had bilateral sensory and motor neuropathy in the lower limbs. A literature review of 40 patients defined the neurotoxicity as "long-lasting" if symptoms persisted for more than 2 months after the discontinuation of lithium (13). However, there are no case reports or case studies of how long the syndrome could last.

Recommendations regarding treatment in such cases include adequate hydration, correction of electrolyte imbalance, extracorporeal removal of lithium by HD (14). Although early HD is recommended as the treatment of choice, no sustainable evidence has been found to prove that it prevents permanent neurologic sequelae. Ristic et al has elaborated how the lithium level of $1.46 \mathrm{mmol} / \mathrm{L}$ in a 63-year-old male produced nephrotoxicity that led to severe renal insufficiency and neurological symptoms. This case report also explains that after HD due to fast redistribution plasma values almost reach the level before dialysis known as rebound effect and often require redialysis as happened in our patient after the first session of HD (15).

A recent study in 2015 in US adults by Olfson attributed 90000 emergency visits to adverse reactions of psychiatric medications, $4.1 \%$ of which was associated with lithium salts alone (16). A high index of suspicion for toxicity is required in patients presenting with neurological features with a baseline value in the normal therapeutic range specifically in elderlies with polypharmacy or renal derangements as early initiation of treatment might prevent permanent neurological damage.

\section{Conclusion}

Unintended lithium toxicity can occur especially in elderlies owing to its narrow therapeutic window and numerous drug interactions. Despite treatment after acute lithium toxicity, some patients might experience persistent symptoms including SILENT. Need is to call for strict adherence to guidelines before prescribing these drugs with potential disabling adverse effects. Dispensing adequate information to patients with an advice to maintain a record book carrying details of renal, thyroid functions and lithium levels can be paramount in prevention of such adversities.

\section{Authors' contributions}

JA: Concept, design, intellectual content, literature search, data acquisition, manuscript preparation, editing, and review. ZM: Data acquisition, design, intellectual content, literature search, manuscript editing, and review. BK: Intellectual content, literature search, data acquisition, manuscript editing and review. YSC: Literature search, clinical studies, data acquisition, manuscript preparation, editing, and review. MG: Intellectual content, literature search, data acquisition, manuscript editing and review.

\section{Ethical issues}

Informed consent statement was obtained from the patient 
for the publication of this report.

\section{References}

1. Machado-Vieira R, Manji HK, Zarate CA Jr. The role of lithium in the treatment of bipolar disorder: convergent evidence for neurotrophic effects as a unifying hypothesis. Bipolar Disord 2009; 11 Suppl 2: 92-109. doi: 10.1111/j.13995618.2009.00714.x.

2. Arancibia A, Corvalan F, Mella F, Concha L. Absorption and disposition kinetics of lithium carbonate following administration of conventional and controlled release formulations. Int J Clin Pharmacol Ther Toxicol 1986; 24(5): 240-5.

3. Cameron P, Little M, Mitra B, Deasy C. Textbook of Adult Emergency Medicine. 5th ed. Edinburgh: Churchill Livingstone; 2000. p. 753-5.

4. Ivkovic A, Stern TA. Lithium-induced neurotoxicity: clinical presentations, pathophysiology, and treatment. Psychosomatics 2014; 55(3): 296-302. doi: 10.1016/j. psym.2013.11.007.

5. Haussmann R, Bauer M, von Bonin S, Grof P, Lewitzka U. Treatment of lithium intoxication: facing the need for evidence. Int J Bipolar Disord 2015; 3(1): 23. doi: 10.1186/ s40345-015-0040-2.

6. Baird-Gunning J, Lea-Henry T, Hoegberg LCG, Gosselin S, Roberts DM. Lithium poisoning. J Intensive Care Med 2017; 32(4): 249-63. doi: 10.1177/0885066616651582.

7. Mégarbane B, Hanak AS, Chevillard L. Lithium-related neurotoxicity despite serum concentrations in the therapeutic range: risk factors and diagnosis. Shanghai Arch Psychiatry 2014; 26(4): 243-4. doi: 10.3969/j.issn.10020829.2014.04.009.
8. Foulser P, Abbasi Y, Mathilakath A, Nilforooshan R. Do not treat the numbers: lithium toxicity. BMJ Case Rep 2017; 2017. doi: 10.1136/bcr-2017-220079.

9. Peng J. Case report on lithium intoxication with normal lithium levels. Shanghai Arch Psychiatry 2014; 26(2): 1034. doi: 10.3969/j.issn.1002-0829.2014.02.008.

10. Strayhorn JM Jr, Nash JL. Severe neurotoxicity despite "therapeutic" serum lithium levels. Dis Nerv Syst 1977; 38(2): 107-11.

11. Nguyen L. Lithium II: irreversible neurotoxicity after lithium intoxication. J Emerg Nurs 2008; 34(4): 378-9. doi: 10.1016/j.jen.2008.04.026.

12. Adityanjee, Munshi KR, Thampy A. The syndrome of irreversible lithium-effectuated neurotoxicity. Clin Neuropharmacol 2005; 28(1): 38-49. doi: 10.1097/01. wnf.0000150871.52253.b7.

13. Schou M. Long-lasting neurological sequelae after lithium intoxication. Acta Psychiatr Scand 1984; 70(6): 594-602. doi: 10.1111/j.1600-0447.1984.tb01254.x.

14. Decker BS, Goldfarb DS, Dargan PI, Friesen M, Gosselin S, Hoffman RS, et al. Extracorporeal treatment for lithium poisoning: systematic review and recommendations from the EXTRIP workgroup. Clin J Am Soc Nephrol 2015; 10(5): 875-87. doi: 10.2215/cjn.10021014.

15. Ristic DI, Siapera MS, Jovic J, Marjanovic VS, Radovanovic M, Fountoulakis KN. Unrecognized acute lithium toxicity: a case report. Cent Eur J Med 2012; 7(6): 700-3. doi: 10.2478/ s11536-012-0058-0.

16. Olfson M. Surveillance of adverse psychiatric medication events. JAMA 2015; 313(12): 1256-7. doi: 10.1001/ jama.2014.15743. 